\title{
§ 9 Fazit des zweiten Teils: Verstärkte Kontrolle als Begleiterscheinung intensivierter Interaktion
}

Zahlreiche Beispiele belegen, dass innerstaatliche Gerichte zu wichtigen Partnern der Menschenrechtsgerichte geworden sind und in fast allen erdenklichen Situationen zur Umsetzung ihrer Entscheidungen beitragen - und dies, obwohl ihnen diese Aufgabe ursprünglich nicht zugedacht war. Dies zeigt, dass die Ansicht, wonach die Umsetzung internationaler Entscheidungen primär eine politische Aufgabe ist, endgültig überholt ist. Wie die Untersuchung zeigt, sind nationale Gerichte heute bereit, in Umsetzung von Entscheidungen der Menschenrechtsgerichte ihre eigene Rechtsprechung anzupassen, die Rechtskraft von Urteilen zu durchbrechen oder gar Gesetze aufzuheben - und dies auch dann, wenn sich das innerstaatliche Recht nicht zum Status internationaler Entscheidungen in der nationalen Rechtsordnung äußert. Nationale Gerichte kompensieren damit bis zu einem gewissen Grad die fehlenden Durchsetzungsmechanismen auf internationaler Ebene und verhelfen den Entscheidungen des EGMR bzw. IAGMR auf der innerstaatlichen Ebene zur tatsächlichen Geltung. Und nicht nur dies: Viele Gerichte sind dazu übergegangen, im Rahmen der Auslegung innerstaatlichen Rechts vorausschauend nach Straßburg bzw. San José zu blicken, um die Rechtsordnung bereits präventiv in Einklang mit den menschenrechtlichen Anforderungen zu bringen und eine „Verurteilung“ des eigenen Staates abzuwenden.

$\mathrm{Zu}$ dieser Entwicklung beigetragen hat sicherlich die Rechtsprechung der Menschenrechtsgerichte selbst, wie sie in Reichweite und Tiefe alle Erwartungen übertroffen hat. Zudem haben beide Gerichte - in unterschiedlichem Maße - Techniken entwickelt, um die Schlagkraft ihrer Entscheidungen auf der innerstaatlichen Ebene zu erhöhen. Im europäischen System hat dies auch zu einer Verrechtlichung des Prozesses der Urteilsüberwachung geführt, der herkömmlicherweise als politischer Prozess in den Händen eines politischen Organes, des Ministerkomitees, galt. Eine wichtige Rolle haben dabei zweifelsohne auch die betroffenen Individuen gespielt, die ein großes Interesse an der tatsächlichen Durchsetzung zu ihren Gunsten ergangener Entscheidungen haben. Oft sind innerstaatliche Gerichte gar die einzige Instanz, an die sie sich wenden können. Die Tatsache, dass Individuen eine so zentrale 
Rolle spielen und die Entwicklung in den Menschenrechtssystemen wesentlich vorangetrieben haben, unterscheidet diese sicherlich auch von Völkerrechtsgebieten, in denen Individuen eine weniger direkte Bedeutung zukommt.

Ausschlaggebend dafür, dass Gerichte aktiv werden und teils auch trotz entgegenstehendem innerstaatlichen Recht Entscheidungen durchsetzen, sind dabei regelmäßig nicht formelle Kriterien. Zwar haben zahlreiche Gerichte absolute Grenzen der Befolgbarkeit internationaler Entscheidungen herausgearbeitet, wozu für viele die Verfassug bzw. ein Verfassungskern zählt. ${ }^{1}$ Allerdings kommt es in der Praxis kaum zu echten Konflikten mit der Verfassung, was sicherlich mit der Ähnlichkeit zwischen Konventionen und Verfassungstexten zusammenhängt. Anders als für die Bestimmung der unmittelbaren Anwendbarkeit von Völkervertragsrecht haben Gerichte auch keinen eigentlichen Test der direkten gerichtlichen Durchsetzbarkeit internationaler Entscheidungen entwickelt. Vielmehr beziehen sie eine flexible Position, indem sie regelmäßig eine Abwägung der verschiedenen Interessen im Einzelfall vornehmen. Ausschlaggebend im Rahmen dieser Abwägung sind dabei inhaltliche Faktoren wie die Schwere der in Rede stehenden Konventionsverletzung und deren möglicherweise andauernden Folgen. Auch die Tatsache, dass sie quasi der letzte Ausweg für die Betroffenen sind, weil die an sich zuständigen Behörden die Umsetzung einer Entscheidung versäumt haben, hat Gerichte zum Einschreiten bewogen.

Dabei hat sich auch gezeigt, dass Gerichte in Europa und Lateinamerika von ganz ähnlichen Erwägungen leiten lassen. Allerdings gibt es durchaus auch Unterschiede zwischen den Systemen. Diese sind aber eher gradueller denn prinzipieller Natur: Gerichte im interamerikanischen System sind gegenüber dem IAGMR ein Stück weit offener als die untersuchten europäischen Gerichte. Die Beispiele legen nahe, dass die weitreichenden Wirkungen, die Gerichte im interamerikanischen Kontext Entscheidungen des IAGMR gewähren, auch mit der Funktion zusammenhängen, welche dieser in der Region wahrnimmt. Viele Gerichte sehen im IAGMR nicht nur einen wichtigen Akteur im Rahmen der Vergangenheitsbewältigung, sondern erkennen diesem auch eine bedeutende Rolle bei der Konsolidierung der noch jungen Demokratien zu. Das Konventionssystem fungiert quasi als externer „Garant" für Rechtsstaatlichkeit.

Die sehr offene Haltung vieler Gerichte lässt sich somit sicherlich auch damit erklären, dass Staaten, die sich in Übergang zu Demokratien befinden, ein größeres Interesse daran haben, Offenheit gegenüber dem Völkerrecht zu signalisieren als etablierte Akteure. ${ }^{2}$ Sadurski hat für Europa aufgezeigt, dass zahlreiche Gerichte in Osteuropa sich mit dem EGMR ,,verbündet“ haben und ,strategische Partnerschaften" eingegangen sind, um zum einen die Rechtsstaatlichkeit abzusichern, zum anderen aber auch ihre eigene Position zu stärken: Dank dem EGMR stünden sie den

\footnotetext{
${ }^{1}$ Siehe dazu $\S 6$.

${ }^{2}$ Alexandra Huneeus, Compliance with Judgments and Decisions, in Cesare Romano et al. (Hrsg.), The Oxford Handbook of International Adjudication (Oxford: Oxford University Press 2014), 437-463, 456. Huneeus bezeichnet dies als ,,politischen Faktor“ der Urteilsbefolgung.
} 
politischen Gewalten nicht mehr „einsam und hilflos“ gegenüber. ${ }^{3}$ Sadurski konnte so zeigen, dass in jungen Demokratien die oft selbst noch jungen Gerichte rezeptiver gegenüber dem EGMR sind, während sich die Gerichte aus den ,alten“ EMRK-Staaten, die teilweise eine lange Grundrechtstradition kennen, ungern von den Straßburger Richtern belehren ließen. ${ }^{4}$

Zwar sind die Aussagen der vorliegenden Studie natürlich nicht allgemeingültig, weil das gezeichnete Bild notwendigerweise unvollständig bleiben muss. So konnte aus sprachlichen Gründen etwa Brasilien nicht berücksichtigt werden, dessen oberstes Gericht für seinen kritischen Kurs gegenüber dem IAGMR bekannt ist. ${ }^{5}$ Auch andere Gerichte in Lateinamerika sind nicht immer nur offen und völkerrechtsfreundlich: Das oberste argentinische Gericht, bislang einer der wichtigsten Partner des IAGMR, scheint in jüngerer Zeit von seinem offenen Kurs abzukommen und nimmt eine zurückhaltendere Position ein. ${ }^{6}$ Andere Gerichte der Region wiederum sind dem IAGMR gegenüber richtiggehend feindlich gesinnt, so etwa das oberste venezolanische Gericht, das seiner Regierung gar den Rückzug aus dem System nahelegte, der bekanntlich inzwischen auch vollzogen ist. ${ }^{7}$ Nicht zuletzt erleidet der IAGMR immer wieder Rückschläge; nicht alle Staaten haben etwa seine Rechtsprechung zu den Amnestien gleich wohlwollend akzeptiert wie die hier dargestellten, ${ }^{8}$ und insgesamt ist die Befolgungsrate der Urteile im interamerikanischen System eher tief. ${ }^{9}$

Dennoch sind die Parallelen insgesamt nicht von der Hand zu weisen. So bestätigt und verdeutlicht die vorliegende Untersuchung auch, dass es noch ,jüngeren“ Gerichten grundsätzlich leichter fällt, Einfluss von außen zu akzeptieren. Ihnen dienen die Menschenrechtsgerichte nicht zuletzt dazu, ihre eigene Stellung zu konsolidieren. Etablierte Gerichte nehmen diese demgegenüber eher als Konkurrenten

\footnotetext{
${ }^{3}$ Wojciech Sadurski, Partnering with Strasbourg: Constitutionalisation of the European Court of Human Rights, the Accession of Central and East European States to the Council of Europe, and the Idea of Pilot Judgments, Human Rights Law Review 9 (2009), 397-453, 438. Siehe zur Legitimierungsfunktion der Öffnung der Rechtsordnung für das Völkerrecht im osteuropäischen Kontext grundlegend Mahulena Hofmann, Von der Transformation zur Kooperationsoffenheit? Die Öffnung der Rechtsordnungen ausgewählter Staaten Mittel- und Osteuropas für das Völker- und Europarecht (Berlin/Heidelberg: Springer 2009).

${ }^{4}$ Sadurski, Partnering with Strasbourg, HRLR.

${ }^{5}$ Siehe dazu Tom Gerald Daly, Brazilian ,Supremocracy ${ }^{\text {and }}$ the Inter-American Court of Human Rights: Unpicking an Unclear Relationship, in Pedro Fortes et al. (Hrsg.), Law and Policy in Latin America. Transforming Courts, Institutions, and Rights (London: Palgrave Macmillan 2017), 3-20. ${ }^{6}$ Siehe dazu ausführlicher $\S 8$ unter 1 .

${ }^{7}$ Siehe Medienmitteilung der IAKMR vom 10. September 2013, IACHR Deeply Concerned over Result of Venezuela's Denunciation of the American Convention, einsehbar unter http://www.oas. org/en/iachr/media_center/preleases/2013/064.asp (zuletzt besucht am 22. September 2019).

${ }^{8}$ So haben Brasilien, Uruguay und Chile ihre Amnestiegesetze anders als Argentinien erst aufgehoben, als der IAGMR diese explizit für konventionswidrig erklärte. In Uruguay folgte auch dann noch ein längeres Hin- und Her zwischen San José und innerstaatlichen Organen. Siehe dazu IAGMR, Case of Gelman v. Uruguay, Monitoring Compliance with Judgment, Anordnung vom 20. März 2013.
}

${ }^{9}$ Siehe zur Befolgung $\S 1$ unter 1.3. 
wahr. Nicht zuletzt dürfte zuweilen ein gewisser (nationaler) Stolz eine Rolle spielen: In Europa wird immer wieder vorgebracht, dass der Einfluss der EMRK unter der Dynamik des EGMR von den ursprünglichen Mitgliedern grundlegend unterschätzt wurde. ${ }^{10}$ Beispielhaft dafür steht die Haltung des Schweizer Bundesgerichts, das die EMRK anfänglich quasi ignorierte ${ }^{11}$ - man ging in der Schweiz ganz selbstverständlich davon aus, dass der EMRK-Beitritt keinen großen Einfluss auf die schweizerische Rechtsordnung haben würde. ${ }^{12}$ Denn im Hinblick auf den Konventionsbeitritt waren präventiv verschiedene größere Anpassungen vorgenommen worden. ${ }^{13}$ Das Bundesgericht stellte sich in der Folge auf den Standpunkt, dass die EMRK-Garantien nicht weiter gingen als die verfassungsmäßigen Rechte in der Schweiz und prüfte vorrangig eine Verletzung der Bundesverfassung. ${ }^{14}$ In den Worten Hotteliers war die unterschwellige Aussage des Bundesgerichts dabei, dass „la constitution fédérale représente le nec plus ultra en matière de protection des droits individuels, le droit conventionnel et la jurisprudence européenne pouvant du coup demeurer largement ignorés. " 15 Ähnliches lässt sich wohl auch in Italien beobachten, wo das italienische Verfassungsgericht sich in einigen Entscheidungen demonstrativ nicht auf den EGMR stützte, sondern diese allein gestützt auf die Verfassung fällte. ${ }^{16}$ Auch die stillschweigende Befolgung von EGMR-Urteilen, wie sie besonders französische Gerichte praktizieren, dürfte schließlich so zu lesen sein. ${ }^{17}$

Die Zusammenarbeit mit den Menschenrechtsgerichten hat insgesamt also sicherlich zu einer Stärkung der Stellung innerstaatlicher Gerichte beigetragen. In

\footnotetext{
${ }^{10}$ Helen Keller \& Alec Stone Sweet, Assessing the Impact of the ECHR on National Legal Systems, in Helen Keller \& Alec Stone Sweet (Hrsg.), A Europe of Rights. The Imact of the ECHR on National Legal Systems (Oxford: Oxford University Press 2008), 677-710.

${ }^{11}$ Keller argumentiert, dass sich die freundliche Haltung gegenüber der EMRK zu ändern begann, als sich effektive Auswirkungen abzeichneten. Helen Keller, Reception of the European Convention for the Protection of Human Rights and Fundamental Freedoms (ECHR) in Poland and Switzerland, Zeitschrift für ausländisches öffentliches Recht und Völkerrecht 65 (2005), 283-349, 313. ${ }^{12}$ Ibid., 305 .

${ }^{13}$ Es handelte sich v. a. um das fehlende Stimm- und Wahlrecht für Frauen sowie die konfessionellen Ausnahmeartikel. Für einen Überblick siehe Mark E. Villiger, Handbuch der Europäischen Menschenrechtskonvention (EMRK) (Zürich: Schulthess 2. Aufl. 1999), Rn. 26 ff.

${ }^{14}$ Statt vieler Bundesgericht, BGE 109 Ia 273 vom 9. November 1983, E 4a): „Damit reicht der Schutzbereich von Art. 8 Ziff. 1 EMRK in Bezug auf die hier aufgeworfenen Fragen nicht weiter als Art. 36 Abs. 4 BV und das ungeschriebene Verfassungsrecht der persönlichen Freiheit." Ferner BGE 119 II 264 vom 3. März 1993, E. 4.a): „Beruft sich ein Beschwerdeführer für den nämlichen Anspruch gleichzeitig auf ein verfassungsmässiges Recht und auf eine Bestimmung der EMRK, so prüft das Bundesgericht in der Regel zunächst, ob der angefochtene Entscheid gegen die Bundesverfassung verstosse. Gegebenenfalls berücksichtigt es dabei allerdings die Konkretisierung bestimmter Rechtsgrundsätze durch die Konventionsorgane [...].“

${ }^{15}$ Michel Hottelier et al., La Suisse devant la Cour européenne des droits de l'homme (Genf/Zürich/Basel: Schulthess 2. Aufl. 2011), 39.

${ }^{16}$ Giuseppe Martinico, National courts and judicial disobedience to the ECHR: a comparative overview, in Oddný Mjöll Arnardóttir \& Antoine Buyse (Hrsg.), Shifting Centres of Gravity in Human Righs Protection: Rethinking relations between the ECHR, EU and national legal orders (London/New York: Routledge 2016), 59-78, 76.

${ }^{17}$ Siehe dazu $\S 5$ unter 4.1 .
} 
einigen Staaten hat sie gar zu einer Erweiterung des Aufgabenkatalogs von Gerichten geführt. So wurde in der Schweiz aufgrund des Konventionssystems quasi durch die Hintertür die Möglichkeit der Verfassungs- bzw. Konventionalitätskontrolle von Gesetzen eingeführt. ${ }^{18}$ Andererseits hat die intensivierte Interaktion mit den Menschenrechtsgerichten auch dazu geführt, dass innerstaatliche Gerichte dazu übergegangen sind, sich stärker abzugrenzen und deren Entscheidungen einer verstärkten Kontrolle zu unterziehen. Regelmäßig verlangen Gerichte heute, dass Entscheidungen der Menschenrechtsgerichte gewisse Anforderungen erfüllen, um befolgt zu werden; viele erkennen zudem in der Verfassung bzw. einem Kern der Verfassung eine absolute Schranke für die Umsetzbarkeit der Entscheidungen. Zuweilen erinnern diese Entscheidungen an das Exequatur-Verfahren, das ausländische Entscheidungen vor ihrer innerstaatlichen Vollstreckung durchlaufen müssen. Während zahlreiche Gerichte schon früh erklärt haben, dass sie nicht bereit sind den Menschenrechtsgerichten uneingeschränkt zu folgen und gewisse Grenzen der Umsetzbarkeit formuliert haben, scheinen sich viele in jüngerer Zeit stärker auf ihre Rolle als Hüter des nationalen Rechts zu besinnen. Dies hängt sicherlich auch damit zusammen, dass Gerichte sich angesichts der Zunahme internationaler Regulierung gegenüber ihren inzwischen mächtigen internationalen Pendants behaupten wollen und eine aktive Teilnahme im Mehrebenensystem einfordern.

Diese von Gerichten aktiver in Anspruch genommene Kontroll- und Filterfunktion an der Schnittstelle zwischen Rechtsordnungen führt zwar wie gesehen nur selten zu einem eigentlichen Rechtsbruch. In den meisten Fällen schränken Gerichte die über die Befolgungspflicht hinausgehenden Urteilswirkungen ein. Auch wenn dieser heute nicht selten fast aggressiv geführte gerichtliche Dialog damit zuweilen völkerrechtsunfreundlich sein mag, ist er regelmäßig nicht völkerrechtswidrig. Dennoch zeugt diese Entwicklung von einer sich im Wandel befindenden Haltung von Gerichten gegenüber den Menschenrechtsgerichten - und möglicherweise gegenüber dem Völkerrecht insgesamt. Und obwohl das Gesamtbild wie gesehen bei näherem Betrachten weniger dramatisch aussieht und es zu kurz gegriffen schiene, die geänderte Haltung von Gerichten als Zeichen der Krise und Abkehr vom Multilateralismus zu lesen, so macht diese Entwicklung doch eine Frage umso drängender, nämlich die normative Frage, welche Rolle innerstaatliche Gerichte an der Schnittstelle zwischen Rechtsordnungen spielen sollen. Dieser Frage soll nun im folgenden, letzten Kapitel nachgegangen werden.

${ }^{18}$ Ibid. 
Open Access Dieses Kapitel wird unter der Creative Commons Namensnennung 4.0 International Lizenz (http://creativecommons.org/licenses/by/4.0/deed.de) veröffentlicht, welche die Nutzung, Vervielfältigung, Bearbeitung, Verbreitung und Wiedergabe in jeglichem Medium und Format erlaubt, sofern Sie den/die ursprünglichen Autor(en) und die Quelle ordnungsgemäß nennen, einen Link zur Creative Commons Lizenz beifügen und angeben, ob Änderungen vorgenommen wurden.

Die in diesem Kapitel enthaltenen Bilder und sonstiges Drittmaterial unterliegen ebenfalls der genannten Creative Commons Lizenz, sofern sich aus der Abbildungslegende nichts anderes ergibt. Sofern das betreffende Material nicht unter der genannten Creative Commons Lizenz steht und die betreffende Handlung nicht nach gesetzlichen Vorschriften erlaubt ist, ist für die oben aufgeführten Weiterverwendungen des Materials die Einwilligung des jeweiligen Rechteinhabers einzuholen.

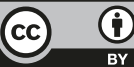

